\title{
Leader Following Consensus of BLDC Motor Speed with Sampling Intervals
}

\author{
Suhaib Masroor, Chen Peng, Zain Anwar Ali, and Muhammad Aamir
}

\begin{abstract}
Permanent Magnet Brushless DC (BLDC) motors have set up diverse uses in industry owing to simple construction, higher efficiency, power density, ease of control as well as enormous torque-to-inertia ratio. Electric Automobiles, Servo Machines (Drives), Aeronautics, Military UAV and inland usages are foremost industrial zone that uses BLDC motors. In this paper, a leader following consensus protocol on sensorless BLDC motor to control the speed using RST pole placement controller is presented. The proposed system model and stimulation is carried out on MATLAB. Simulation results validates the proposed system.
\end{abstract}

Index Terms-Multi-agent system, consensus algorithm, brushless DC motor, RST controller.

\section{INTRODUCTION}

In the preceding years, the study of multi-agent systems (herein after MAS), has received a foremost concentration within thecontrol researchers. A major complexity that appears frequently in the framework of coordination of MAS is the consensus problem.

In Nature, we can observe that there are many phenomena's which possess consensus problem, for example birds motion, fishes schooling, cooperatively relocation of mammals etc. In MAS, every agent has sovereignty to perform desired task but when working as group, they all need to be in settlement on some common interest.

We can classify consensus problems as leaderless and leader-following. When group of agents are able to communicate with each other having local information to achieve desired task is said to be leaderless consensus whereas in leader-followingscenario, there is a leader which directs the desired trajectory and all the following agents are required to be connected with leader to achieve desired task.

In leader following MAS, followers don't communicates with each other, they receive information from the leader to reach a consensus. Mostly, leaders are sovereign to its followers but effects the followers so that controlling the leader leads to fulfill desired task. The advantage of this methodology is that a leader defines the reference trajectory and formation stability is inferred by the individual agents control protocol. Jadbabaie et al in [1], proved that the states of following agents will converge to that of leader with the

Manuscript received January 30, 2016; revised March 28, 2016.

Suhaib Masroor and Chen Peng is with School of Mechatronics Engineering and Automation, Shanghai University, Shanghai, China (email: suhaibmasroor1@gmail.com, c.peng@shu.edu.cn).

Zain Anwar Ali is with College of Automation Engineering, Nanjing University of Aeronautics and Astronautics. Nanjing, China (e-mail: zainanwar86@hotmail.com). passage of time, as long as following agents are connected with leader. In [2], consensus problem for fixed and switching topologies leader-following higher order MAS having linear dynamics is considered using local information. Algebraic graph theory, Riccati and Lyapunov inequalities are used for system design. Mahmoud in [3], consider discrete consensus of leader following MAS with fixed and switching topologies subjected to disturbances. A controller based on dynamic output feedback is designed that satisfies $\mathrm{H} \infty$ routine. Consensus of multi-agent systems is proved by feedback gain matrix derived by solving LMI's.

In several application, agents may not require to exchange information continuously instead information exchange occurs as discrete sampling intervals. Therefore, it is necessary to consider the case of sampled data controlled consensus algorithms thereby attaining robustness, flexibility and reduction in cost. Some fruitful sampled data control algorithms are addressed in [4]-[8]. In [9], Ding address the consensus problem of sampled data leader following nonlinear MAS with communication delay. Due todelay, adjustable sampling period control protocol is proposed thereby converting it into stability problem and derive a delay dependent stability criteria by using Lyapunov-Krosovskii functional method and solve a set of LMI to obtain controller gain. Qingsong in [10], considered the sampled data leader following consensus for second order MAS with fixed and switching topologies.

A leader following tracking control problem occurs when agents are required to follow a desired path. In [11], Wang studies tracking control of a discrete time leader-following consensus of group of agents under fixed and switching topology. Since active leader's velocity is assumed to be unknown, a neighbor's information and state estimation rule is used for each agent to track the leader. Sufficiency conditions are derived by using the graph theory, matrix theory and Lyapunov theorem which prove the stability of system. In [12], problem of consensus tracking for single and double integrator leader following multi agent system in a scenario of communication failure is considered. In case of directed fixed topology, a sufficiency condition to reduce tracking error is derived.

Above contemplations encouraged us to investigate the leader-following consensus problem for Brushless dc motor (herein after BLDC) connected in leader following manner with sampled intervals. The BLDC is a Synchronous motors in which winding are on stator side and permanent magnet at the rotor side. BLDC motor exhibits linear relationship between current and torque, voltage and rpm. BLDC motors have been widely used in automotive (hybrid vehicles), computers, household products and especially in aerospace application [13]. When compared with brushed DC motor or 
induction motors, BLDC have numerous advantages including linear torque-speed characteristics, higher speed ranges, noiseless operation, high dynamic response and efficiency, good control over driving system. The torque generated by motor and speed of motor depends on magnetic field generated by motor winding, which itself rely on flow of current. Therefore, the speed of motor can be controlled by controlling the rotor voltages and current [14]. There are various methods for controlling the speed and torque of BLDC motors. Mostly, P, PI and PID controller are used for controlling motor speed [15].

In this paper, a control proposal for consensus of multiple BLDC motor based MAS using RST controller is presented, so that all the motors converges to common trajectory i-e speed, defined by a leader. The communication topology is supposed to be fixed.

The paper is organized as follows. Section II contain background of graph theory as well as concept of consensus in multi agent leader following scenario.In section III, we explore the dynamic model of BLDC motors. Section IV shows the analysis and design of proposed controller with the model obtained in section III. In Section V we present the application of the consensus protocol to the problem. Section VI shows the stimulation results and finally conclusion is given.

\section{PRELIMINARIES AND GRAPH THEORY}

Agents can achieve their collective goal by sharing their information with each other with the help of communication network. This exchange of information between agents can be demonstrated using graphs. A graph $G(V, E)$, consist of a node set $V=\left\{v_{1}, \ldots v_{N}\right\}$, and $E \subseteq V \times V$ an edge set. The edge $\left(v_{i}, v_{j}\right)$ represents that $v_{i}$ is a parent node and $v_{j}$ is a child node that obtains information from $v_{i}$. The neighbors of agent $v_{i}$ is represented as $N_{i}$, so we can say that $v_{j}$ is a neighbor of $v_{i}$. The adjacency matrix $\quad \Xi=\left[\mathfrak{a}_{i j}\right] \in \mathbb{R}^{N \times N}$ is $\mathfrak{a}_{i i}=0, \mathfrak{a}_{i j}$ is a positive value if $\left(v_{j}, v_{i}\right) \in E$ and $\mathfrak{a}_{i j}=0$ otherwise. For undirected graph the only difference in adjacency matrix is that $\mathfrak{a}_{i j}=\mathfrak{a}_{j i}$ for all $i \neq j$. The Laplacian matrix $L=\left[L_{i j}\right] \in \mathbb{R}^{N \times N}$ is $L=\Delta-\Xi$, where $\Delta=$ $\operatorname{diag}\left\{d_{1}, \ldots d_{N}\right\}$ is the degree matrix. The Laplacian $L$ of the graph $G$ is a matrix such that $L_{i i}=\sum_{j \neq i} \mathfrak{a}_{i j}$ and $L_{i j}=$ $-a_{i j}, i \neq j$. The row sum of $L$ is zero with 0 simple eigenvalue having eigenvector of $\mathbf{1}$.

Lemma 1. In [16], for an undirected connected graph, $L$ has simple zero eigenvalue and smallest nonzero eigenvalue $\lambda_{2}$ of $L$ satisfies $\lambda_{2}=\min _{x \neq 0,1^{T} x=0} \frac{x^{T} L x}{x^{T} x}$

Lemma 2. In [16], for strongly connected $G$, there exists a left eigenvector $r=\left[r_{1}, \ldots, r_{N}\right]^{T}$ of $L$ having zero eigenvalue and $R L+L^{T} R \geq 0$, where $\quad R=$ $\operatorname{diag}\left(r_{1}, \ldots, r_{N}\right)$.

For connected graph, algebraic connectivity is smallest nonzero eigenvalue $\lambda_{2}$ of associated Laplacian matrix $L$.

Here, node 0 represents the leader, it exchange information only with those agents which are in its neighborhood. In this way, we have another graph $\bar{G}$ containing node 0 , the graph $G$ and the edges representing the information exchange between node 0 and its neighbors. In this type of network, we are going to design a controller $u_{i}, i=1,2, \ldots, N$ to make sure that all the following agents must follow the leader.

Definition: For every agent $i \in\{1, \ldots, N\}$, consensus is thought to be reached for any initial condition $x_{i}(0), i=$ $1, \ldots, N$ in leader following scenario such that there exists a feedback $u_{i}$ of $\left\{x_{j}: j \in N_{i}\right\}$ satisfies $\lim _{t \rightarrow \infty} \| x_{i}(t)-$ $x o(t)=0, i=1, \ldots ., N$.

\section{DYNAMIC MODEL OF BLDC MOTORS}

In BLDC motors, the rotor rotates by the action of rotating magnetic field produced by various electronic drive systems. A back EMF is generated in stator winding when a rotating magnet pass over the stator pole. As seen in the diagram, star connected BLDC motor uses six step inverter to provide three phase supply for stator coils to generate rotating magnetic field. The dynamic model of star connected BLDC motor (Fig. 1) is given by [13], [14].

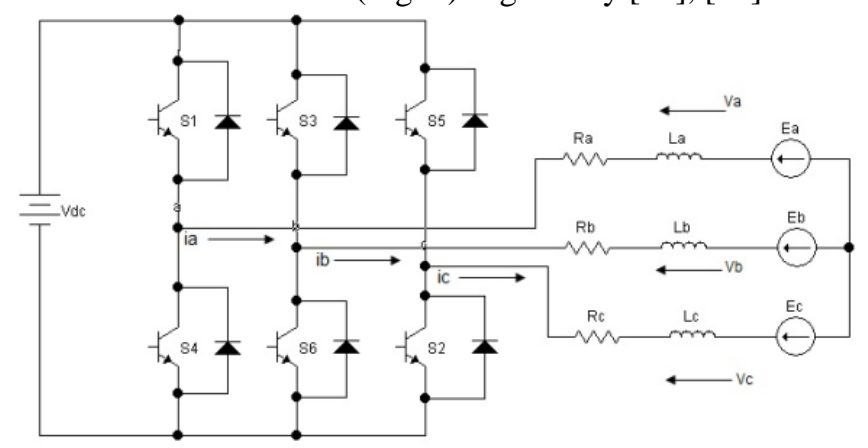

Fig. 1. Dynamic model of BLDC motor.

$$
\begin{aligned}
& \vartheta_{a}=\Omega_{a} i_{a}+H \frac{d i_{a}}{d t}+\varrho_{a} \\
& \vartheta_{b}=\Omega_{b} i_{b}+H \frac{d i_{b}}{d t}+\varrho_{b} \\
& \vartheta_{c}=\Omega_{c} i_{c}+H \frac{d i_{c}}{d t}+\varrho_{c} \\
& {\left[\begin{array}{l}
\vartheta_{a} \\
\vartheta_{b} \\
\vartheta_{c}
\end{array}\right]=} \\
& {\left[\begin{array}{ccc}
\Omega_{a} & 0 & 0 \\
0 & \Omega_{b} & 0 \\
0 & 0 & \Omega_{c}
\end{array}\right]\left[\begin{array}{l}
i_{a} \\
i_{b} \\
i_{c}
\end{array}\right]+} \\
& {\left[\begin{array}{ccc}
\mathrm{H}_{a} & \Phi_{a b} & \Phi_{a c} \\
\Phi_{b a} & \mathrm{H}_{b} & \Phi_{b c} \\
\Phi_{c a} & \Phi_{c b} & \mathrm{H}_{c}
\end{array}\right] \frac{d}{d t}\left[\begin{array}{l}
i_{a} \\
i_{b} \\
i_{c}
\end{array}\right]+\left[\begin{array}{l}
\varrho_{a} \\
\varrho_{b} \\
\varrho_{c}
\end{array}\right]}
\end{aligned}
$$

It is assumed that motor is loss free i-e neither iron losses nor saturated, stator resistances are same, zero mutual inductances and constant self-inductances.

$$
\Omega_{a}=\Omega_{b}=\Omega_{c}=\Omega
$$




$$
\begin{aligned}
& H_{a}=H_{b}=H_{c}=H \\
& \Phi_{a b}=\Phi_{a c}=\Phi_{b a}=\Phi_{b c}=\Phi_{c a}= \\
& \Phi_{c b}=\Phi=0 \\
& {\left[\begin{array}{l}
\vartheta_{a} \\
\vartheta_{b} \\
\vartheta_{c}
\end{array}\right]=} \\
& {\left[\begin{array}{ccc}
\Omega & 0 & 0 \\
0 & \Omega & 0 \\
0 & 0 & \Omega
\end{array}\right]\left[\begin{array}{l}
i_{a} \\
i_{b} \\
i_{c}
\end{array}\right]+\quad\left[\begin{array}{ccc}
\mathrm{H} & 0 & 0 \\
0 & \mathrm{H} & 0 \\
0 & 0 & \mathrm{H}
\end{array}\right] \frac{d}{d t}\left[\begin{array}{l}
i_{a} \\
i_{b} \\
i_{c}
\end{array}\right]+\left[\begin{array}{l}
\varrho_{a} \\
\varrho_{b} \\
\varrho_{c}
\end{array}\right]} \\
& {\left[\begin{array}{l}
\vartheta_{a} \\
\vartheta_{b} \\
\vartheta_{c}
\end{array}\right]=\Omega\left[\begin{array}{l}
i_{a} \\
i_{b} \\
i_{c}
\end{array}\right]+\mathrm{H} \frac{d}{d t}\left[\begin{array}{l}
i_{a} \\
i_{b} \\
i_{c}
\end{array}\right]+\left[\begin{array}{l}
\varrho_{a} \\
\varrho_{b} \\
\varrho_{c}
\end{array}\right]}
\end{aligned}
$$

Back EMF is given by,

$$
\begin{array}{r}
\varrho_{a}=\omega_{r} \cdot K_{r} f\left(\theta_{r}\right) \\
\varrho_{b}=\omega_{r} \cdot K_{r} f\left(\theta_{r}-\frac{2 \pi}{3}\right) \\
\varrho_{c}=\omega_{r} \cdot K_{r} f\left(\theta_{r}+\frac{2 \pi}{3}\right)
\end{array}
$$

Such that $\omega_{r}$ is angular rotor velocity, $\theta_{r}$ is the rotor position and $K_{r}$ is the rotor magnetic flux constant. Torque generated by the motor is

$$
T_{e}=\frac{\varrho_{a} i_{a}+\varrho_{b} i_{b}+\varrho_{c} i_{c}}{\omega_{r}}
$$

By considering friction co-efficient $B$, inertia $J$ and loaded torque $T_{L}$, than the generated torque is given as

$$
T_{e}=T_{L}+J \frac{d \omega_{r}}{d t}+B \omega_{r}
$$

\section{Proposed MOdel FOR SPEEd CONTROL OF BLDC MOTORS}

RST is the sophisticated pole placement centered controller consist of $\mathrm{R}, \mathrm{S} \& \mathrm{~T}$ polynomials tuned by pole placement. In [17], a Robust RST controller is used to control the Euler angles of elevation and velocities of a TriCopter UAV. In [18], it is shown that Vehicle and Lane tracking under automated highway system are made by RST controller.

The advantage of designing RST controller using pole placement approach allow us to lift up the restriction on system zeros, on time delay and degree of polynomials $A$ and $B$ of the system. The response of the system to be controlled is described by the transfer function $B / A$ while the desired system response or reference model is given as
$B_{m} /_{A_{m}}$. Fig. 2 shows the close loop structure of the RST controlled system [19].

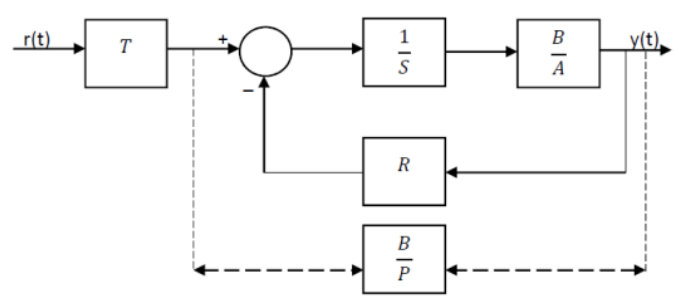

Fig. 2. Pole placement with RST controller.

The open loop transfer function is given as

$$
H_{o l}=\frac{B R}{A S}
$$

The close loop response of the system is given by

$$
H_{c l}=\frac{B}{P}
$$

As seen, closed loop poles are defined by polynomial $P$ which play crucial role for the regulation performance. The polynomial $P$ can be specified directly to achieve desired performance. Now to compute $\mathrm{R}$ and $S$, following equation, known as "Diophantine equation" must be solved

$$
\begin{aligned}
P & =A S+B R \\
H_{c l} & =\frac{B}{A S+B R}
\end{aligned}
$$

Tracking problem occurs when we want the output of the system to track reference signal. The close loop transfer function with tracking is given as

$$
H_{c l}=\frac{T B}{A S+B R}
$$

Ideally, it is required that output of the system tracks the desired path or trajectory even if there is any change in the reference. The transfer function of reference model, determined from desired performance, is given as

$$
H_{m}=\frac{B_{m}}{A_{m}}
$$

whereas the reference trajectory model is given as

$$
y^{*}=\frac{B_{m}}{A_{m}} r(t)
$$

This concludes the complete RST system model as shown in Fig. 3.

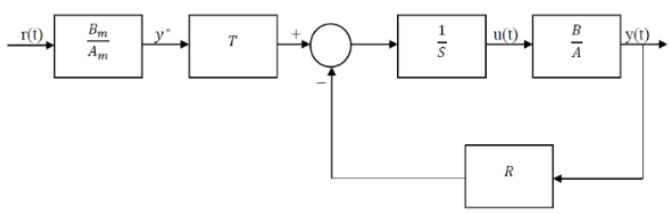

Fig. 3. Pole placement, tracking and regulation.

The general scheme of BLDC motor speed control is shown in Fig. 4. 


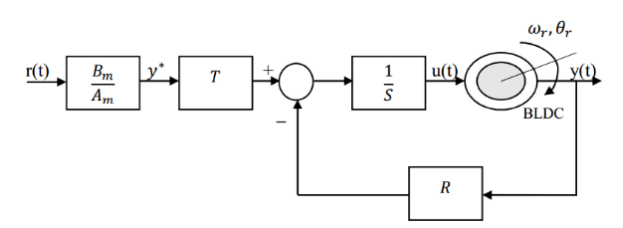

Fig. 4. Schematic of RST based BLDC motor speed control.

Now consider the response of $i_{t h}$ agent, our strategy is to use pole placement RST controller for consensus on speed of $i_{\text {th }}$ agent such that $B_{m} / A_{m}$ is desired response and $B / A$ is the actual response of the agent. With different sampling intervals for agents, we assumed that this reference trajectory is dictated by the leader and for convergence to common trajectory all the agents need to be connected with the leader.

\section{CONSENSUS PROTOCOL FOR BLDC MOTORS}

In this paper, a leader following consensus problem for team of $n$ agents (BLDC motors) is considered, so that all the motors converges to common speed defined by a leader as $\omega_{\text {ref }}$. Moreover, it is also supposed that the information exchange between the leader and follower is adequately interconnected. Since the following agents are not cognizant of desired speed, therefore to reach appropriate $\omega_{\text {ref }}$ they must keep connected. The control input for agent $i$ is defined as

$$
u_{i}(t)=-\sum_{k \in N_{i}}\left(\omega_{i}-\omega_{k}\right)+\left(\omega_{r e f}-\omega_{i}\right)
$$

\section{Results}

The results of the preceding sections are demonstrated through computer simulations.

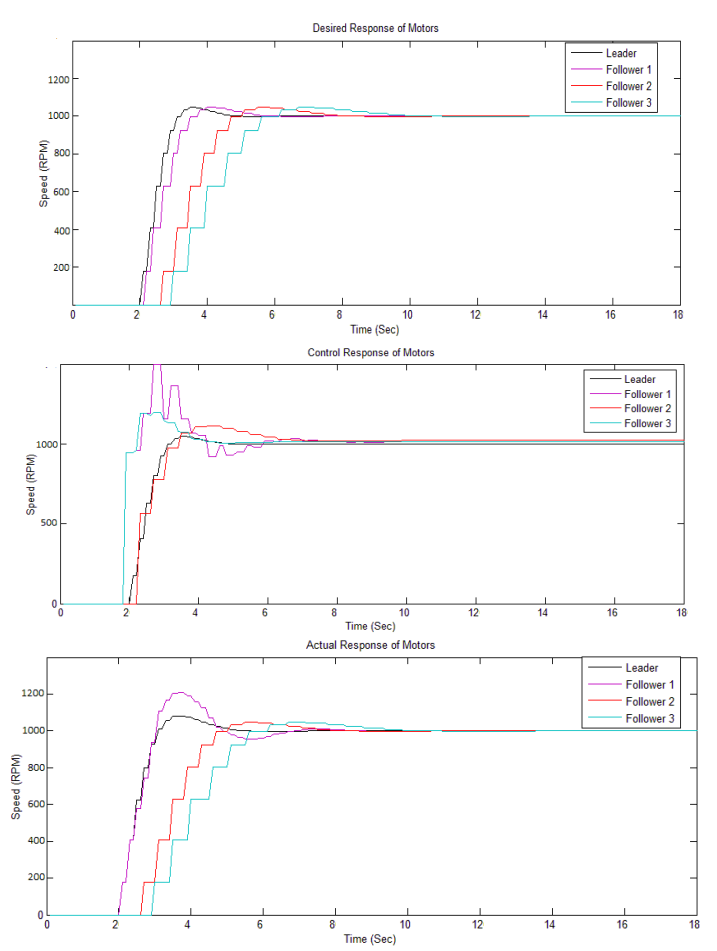

Fig. 5. Validation of consensus algorithm. (a) Desired response of leader along with followers, (b) Individual agent's controller response, (c) Actual response of leader and following agents.
As seen in the graphs, the following agents having random sampling intervals converge to the common speed defined by the leader as time goes on.

\section{CONCLUSION}

In this paper, we study the application of leader following consensus algorithm on BLDC motor. By employing RST controller, we have shown that all the motors initialized at different intervals will ultimately converge to the desired speed defined by the leader as time goes on. The use of consensus algorithm provide robust methodology for industrial applicationsbut in future more research efforts should be focused in this direction.

\section{ACKNOWLEDGEMENT}

This work is supported by National Natural Science Foundation of China under grant 61273114, the Innovation Program of Shanghai Municipal Education Commission under grant 14ZZ087, the Pujiang Talent Plan of Shanghai City China under grant 14PJ1403800, the International Corporation Project of Shanghai Science and Technology Commission under grants 14510722500, 15220710400.

\section{REFERENCES}

[1] A. Jadbabaie, J. Lin, and A. S. Morse, "Coordination of groups of mobile autonomous agents using nearest neighbor rules," IEEE Transactions on Automatic Control, vol. 48, no. 6, 2003.

[2] W. Ni and D. Z. Cheng, "Leader following consensus of multi-agent systems under fixed and switching topologies," Systems and Control Letters, vol. 59, 2010, pp. 209-217

[3] M. S. Mahmoud and G. D. Khan, "Leader-following discrete consensus control of multi-agent systems with fixed and switching topologies," Journal of the Franklin Institute, vol. 352, 2015, pp. 2504-2525.

[4] T. Hayakawa, T. Matsuzawa, and S. Hara, "Formation control of multi-agent systems with sampled information — Relationship between information exchange structure and control performance," in Proc. 45th Conf. IEEE Conf. Decision and Control, 2006, pp. 43334338.

[5] H. Liu, G. Xie, and L. Wang, "Necessary and sufficient conditions for solving consensus problems of double-integrator dynamics via sampled control," Int. J. Robust Nonlinear Control, 2010, vol. 20, no. 15, pp. 1706-1722.

[6] Y. Gao and L. Wang, "Consensus of multiple dynamic agents with sampled information," IET Control Theory Appl., 2010, vol. 4, no. 6, pp. 945-956.

[7] Y. C. Cao and R. Wei, "Sampled-data discrete-time coordination algorithms for double-integrator dynamics under dynamic directed interaction," Int. J. Control, 2010, vol. 83, no. 3, pp. 506-515.

[8] Y. C. Cao, R. Wei, and L. Yan, "Distributed discrete-time coordinated tracking with a time-varying reference state and limited communication," Automatica, 2009, vol. 45, no. 5, pp. 1299-1305.

[9] L. Ding, Y. Zhao, and G. Guo, "Sampled-data leader-following consensus of non-linear multi-agent systems with communication delay," 2013.

[10] Q. S. Gong, L. Y. Zhao, and L. Kun, "Leader-following consensus in second-order multi-agent systems with sampled data," in Proc. 31st Chinese Control Conference, 2012, Hefei, China.

[11] F. Wang, L. X. Gao, and L. Y. Wang, "Tracking control for multiagent consensus for discrete-time case," 2011.

[12] H. R. Li, Q. H. Wu, and D. Sabir, "Consensus tracking algorithms with estimation for multi-agent system," 2015.

[13] J. Sriram and K. Sureshkumar, "Speed control of BLDC motor using fuzzy logic controller based on sensor less Technique," in Proc. International Conference on Green Computing Communication and Electrical Engineering, 2014.

[14] A. Stînean, S. Preitl, R. E. Precup, C. A. Dragoş, and M. BogdanRădac, "Hybrid fuzzy control solutions for brushless DC drives with variable moment of inertia," in Proc. IEEE 10th Jubilee 
International Symposium on Intelligent Systems and Informatics, 2012, Subotica, Serbia.

[15] D. Talebikordkandi, H. Khanbabaiegardeshi, and H. Torkaman, "An improved method to control the speed and flux of PM-BLDC motors," in Proc. the 6th International Power Electronics Drive Systems and Technologies Conference, Shahid Beheshti University, Tehran, Iran, 2015.

[16] Z. K. Li and Z. S. Duan, "Cooperative control of multi-agent Systems a consensus region approach," Automation and Control Engineering, 2014.

[17] Z. A. Ali, D. B. Wang, and M. Aamir, "Design a robust RST controller for stabilization of a tri-copter UAV," Pakistan Journal of Engineering Technology and Science, 2015, vol. 5, no. 1, pp. 60-71

[18] Z. A. Ali,, L. Ning, I. H. Farooqui, F. H. Rizvi, and M. Faraz, "Regulation, pole placement and tracking (RST) robust controller for automatic highway system," International Research Journal of Electronics and Communication Engineering, 2015, vol. 1, no. 4.

[19] I. D. Landau and Z. Gianluca, "Digital control systems design, identification and implementation," Communications and Control Engineering, 2006.

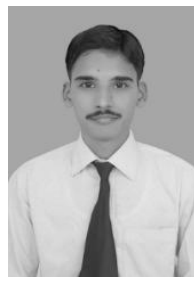

Suhaib Masroor received his B.S degree in biomedical engineering from Sir Syed University of Engineering and Technology, Karachi, Pakistan in 2008 and the M.engg degree in electronics engineering (with specialization in industrial electronics) from NED University of Engineering and Technology, Karachi, Pakistan in 2012. At present doing $\mathrm{PhD}$ in control theory and engineering from Shanghai University, Shanghai, China under supervision of Prof. Chen Peng.

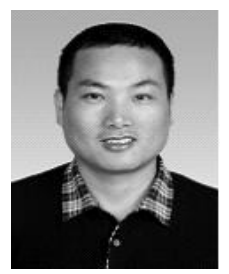

Chen Peng received the B.Sc and M.Sc degree in coal preparation and the $\mathrm{PhD}$ degree in control theorem and control engineering from Chinese University of Mining Technology, Xuzhou, China, in 1996, 1999 and 2002 respectively. From Sep: 2002 to Aug: 2004, he was a postdoctoral research fellow in applied mathematics with Nanjing Normal University, Nanjing, China. From Nov: 2004 to Jan: 2005, he was a research associate with Hong Kong University, Pokfulam, Hong
Kong. From July 2006 to Aug: 2007, he was a visiting scholar with Queensland University of Technology, Brisbane, Australia. From Sep: 2010 to Aug: 2012, he was a postdoctoral research fellow with Central Queensland University, North Rock Hampton, Australia. Presently, he is a professor with School of Mechatronics Engineering and Automation, Shanghai University, Shanghai, China. His research interest includes analysis and synthesis of networked control systems, power systems, fuzzy control systems and interconnected systems.

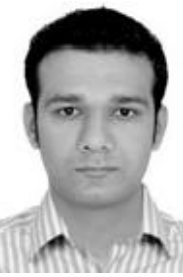

Zain Anwar Ali received his B.S. degree in electronic engineering from the Sir Syed University of Engineering and Technology, Karachi, Pakistan in 2010. And currently doing $\mathrm{PhD}$ in control theory and control engineering from Nanjing University of Aeronautics and Astronautics, Nanjing, China from 2015. He is working as a faculty member and researcher in the Department of Electronic Engineering, Sir Syed University of Engineering and Technology

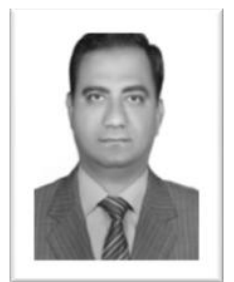

Muhammad Aamir received his B.S. degree in electronic engineering from the Sir Syed University of Engineering and Technology, Karachi, Pakistan in 1998 and the MS degree in electronics engineering (with specialization in telecommunication) from same university in 2002. He accomplished his $\mathrm{PhD}$ in electronics engineering from Mehran University of Engineering and Technology. $\mathrm{He}$ is a life time member of Pakistan Engineering Council and professional member of IEEE for last 12 years. 